\title{
Original Research \\ Coronary angiographic findings in renal transplant patients with chronic coronary artery disease: a pilot study
}

\author{
Guangyao Zhai ${ }^{1}$, Jianlong Wang ${ }^{1}$, Yuyang Liu ${ }^{1}$, Yujie Zhou ${ }^{1}$ ** \\ ${ }^{1}$ Department of Cardiology, Beijing Anzhen Hospital, Capital Medical University, Beijing Institute of Heart Lung and Blood Vessel Disease, Beijing \\ Key Laboratory of Precision Medicine of Coronary Atherosclerotic Disease, Clinical Center for Coronary Heart Disease, Capital Medical University, \\ 100029 Beijing, China \\ *Correspondence: azzyj12@163.com (Yujie Zhou) \\ Academic Editors: Jerome L. Fleg and Giuseppe Coppolino \\ Submitted: 25 October 2021 Revised: 15 January 2022 Accepted: 17 January 2022 Published: 17 February 2022
}

\begin{abstract}
Background: Patients that undergo renal transplantation (RT) often suffer from high rates of cardiovascular disease-related mortality, yet, most of the studies focus on coronary angiography performed as screening to entry in a waiting list and not, as for clinical indication, after renal transplantation. Methods: This study examined coronary angiography findings from 45 patients with functional renal grafts for over 6 months that were analyzed in Anzhen Hospital (Beijing, China) from 2014-2019. For comparison purposes, we additionally examined coronary angiography findings from 45 age- and sex-matched patients undergoing chronic dialysis due to end-stage renal disease (ESRD). We used the SYNTAX score to gauge coronary artery disease (CAD) severity. Results: The duration of ESRD in patients in the RT group was significantly longer than for that of patients in the dialysis comparison group (19.31 \pm 7.83 years vs. $11.43 \pm 8.04$ years, $p<0.001)$. The SYNTAX scores for patients in the dialysis and RT groups were $17.76 \pm 7.35$ and $12.57 \pm 5.61$, respectively $(p<0.01)$. We found that $64.4 \%$ and $28.9 \%$ of dialysis and RT patients, respectively, exhibited the presence of moderate or severe calcified lesions upon examination. In addition, the SYNTAX scores of RT patients were correlated with ESRD duration $(p<$ 0.001). Conclusions: We observed less serious CAD in RT patients relative to long-term dialysis patients even though the former group exhibited a longer mean ESRD duration. Both groups exhibited high rates of calcification of the coronary artery, even following RT.
\end{abstract}

Keywords: renal transplantation; coronary artery disease; SYNTAX score; end-stage renal disease; coronary calcification

\section{Introduction}

Individuals suffering from end-stage renal disease (ESRD) are at an elevated risk of prematurely developing cardiovascular disease (CVD) as a result of altered sodium or fluid homeostasis, atherosclerotic inflammation, and pronounced calcification [1]. ESRD patients with CVD exhibit significant reductions in both their short- and longterm survival.

Renal transplantation (RT) of kidney graft from a healthy donor remains one of the primary viable treatments for ESRD patients suffering from a loss of renal function [2,3]. The rates of RT recipient survival have been steadily rising in recent years owing to advances in patient care and the development of increasingly reliable immunosuppressive drug regimens that can improve graft acceptance. Despite these advances, however, cardiovascular complications remain the leading factor adversely impacting the postoperative survival of RT patients [4,5]. Indeed, RT patients typically exhibit a wide range of cardiovascular risk factors associated with the elevated rates of CVD in this patient population, including general risk factors and additional factors that are more specifically associated with the renal failure and transplantation process [6]. Despite its serious association with mortality in RT patients, there have been insufficient studies conducted to date characterizing postoperative coronary artery disease (CAD) in this patient population. Immunosuppressive drug regimens have been shown to aggravate a number of CAD-related risk factors including diabetes, hypertension, and hyperlipidemia. However, transplantation is also associated with the regression of uremia-associated risk factors. RT patients are also likely to have an extensive history of risk factors including prolonged renal disease, prior transplant rejection, preexisting CVD, and history of dialysis, all of which can increase the risk of CAD development. Once it arises, CAD can be very difficult to treat effectively in RT patients, making it very important that the etiology and manifestation of this condition be better clarified in this at-risk population $[7,8]$. However, recent studies have also shown that RT patients exhibit lower rates of CVD than do patients undergoing long-term dialysis.

The goal of the present study was to characterize angiographic findings associated with CAD in RT patients following kidney transplantation and to compare these findings to those of patients undergoing long-term dialysis. In addition, we sought to identify any correlations between angiographic findings and patient clinicopathological characteristics. 


\section{Methods}

\subsection{Study population}

Approximately 90,000 individuals underwent coronary angiography (CAG) in Anzhen Hospital (Beijing, China) during the study period from 2014-2019. Of these patients, $56(0.06 \%)$ had a history of undergoing RT. Of these patients, we then enrolled individuals that had had a functioning graft for 6 or more months prior to CAG into the present study. When patients had undergone more than one angiographic assessment during the study period, only the initial examination was used for analyses. Based on these criteria, we ultimately identified a group of 45 RT patients that had undergone CAG during this study period.

Over this same study period, 90 ESRD patients undergoing dialysis (hemodialysis or peritoneal dialysis) had also been subjected to CAG at our institution. Following the exclusion of patients that had been undergoing dialysis for $<6$ months, we created a 45 -patient-group that was ageand sex-matched to the RT group for comparison purposes. This study was conducted in accordance with the Declaration of Helsinki and was approved by the Institutional Review Board.

\subsection{Clinical and laboratory data}

For each patient, basic demographic data were collected. In addition, patients were assessed for the presence of CAD-associated risk factors smoking, hyperlipidemia, diabetes, and essential hypertension (EH). Total ESRD and dialysis duration between the time of RT and CAG were also determined. Prior to CAG, plasma creatinine levels were measured in order to estimate glomerular filtration rate (eGFR) based upon the Modification of Diet in Renal Disease equation formula.

$\mathrm{EH}$ and diabetes were diagnosed in patients based upon previously reported criteria [9]. A minimum of two systolic blood pressure readings $\geq 140 \mathrm{mmHg}$ and/or diastolic blood pressure readings $\geq 90 \mathrm{mmHg}$ were considered to be indicative of $\mathrm{EH}$, whereas a fasting blood glucose level of $\geq 7.0 \mathrm{mmol} / \mathrm{L}$, or a random glucose level of $\geq 11.1$ $\mathrm{mmol} / \mathrm{L}$, or a previous diabetes diagnosis was used to identify patients suffering from diabetes. The regular smoking of cigarettes was used to determine whether or not a given patient was a smoker.

\subsection{Angiographic data}

CAG is currently the gold standard approach to diagnosing $\mathrm{CAD}$, and as such two experienced cardiologists reviewed the CAG analyses of all patients in the present study. The SYNTAX scoring system was used to assess coronary lesion severity, and the number of stenotic coronary arteries was similarly assessed. Furthermore, the calcification of identified lesions was classified as being either none/mild or moderate/severe, as discussed in previous studies [10]. Numbers of coronary artery lesions were determined via enumeration of those coronary vessel seg- ments with a diameter of $>2.0 \mathrm{~mm}$ exhibiting $\geq 50 \%$ stenosis, including left main, left anterior descending, left circumflex, right coronary artery, and main branches. Based on these findings, patients were classified into single-, two-, or three-vessel disease groups. In cases where angiographic assessments or SYNTAX scores differed significantly between reviewers, both reviewers assessed the film again and came to a consensus via discussion.

\subsection{Correlations between clinicopathological data and $C A G$ findings}

Both univariate and multivariate analyses were used to assess the relationship between specific clinicopathological variables, SYNTAX scores, and CAD severity in RT patients.

\subsection{Statistical analysis}

Patients in the dialysis and RT groups had been matched according to age and gender distributions, with matching having been conducted without consideration for CAG findings. Continuous variables are given as means \pm standard deviation, while categorical variables are frequencies (percentages).

Student's $t$-tests, Spearman's rank correlation coefficient, chi-squared Pearson correlation tests, and multivariate regression analyses were used to compare values in the present study, with $p<0.05$ as the significance threshold. SPSS v 23.0 (IBM Corp., Chicago, IL, USA) was used for all statistical testing.

\section{Results}

\subsection{Baseline patient characteristics}

The characteristics of patients prior to matching are compiled in Table 1. Those patients in the RT group were significantly younger than were those in the dialysis (57.04 \pm 8.05 years vs. $61.88 \pm 11.36$ years, $p=0.003$ ). In addition, patients in the RT group exhibited higher levels of total cholesterol (TC), triglyceride (TG), low-density lipoprotein cholesterol (LDL-C), high density lipoprotein cholesterol (HDL-C), eGFR and ejection fraction (EF) than did those in the dialysis group.

Following matching, there were no differences in age and family history of CVD distributions between the dialysis and RT groups (Table 2). However, patients in the RT group exhibited higher rates of hyperlipidemia, EH, diabetes, and smoking relative to the dialysis group. RT patients also had a significantly longer total ESRD duration (19.31 \pm 7.83 years) relative to patients in the dialysis group (11.43 \pm 8.04 years). The RT and dialysis group patients also had eGFR values of $69.2 \mathrm{~mL} / \mathrm{min} / 1.73 \mathrm{~m}^{2}$ and 6.4 $\mathrm{mL} / \mathrm{min} / 1.73 \mathrm{~m}^{2}$, respectively. 
Table 1. Baseline characteristics of involved participants before matching.

\begin{tabular}{|c|c|c|c|c|}
\hline & Total & ESRD & RT & \multirow{2}{*}{$p$ value } \\
\hline & $\mathrm{n}=146$ & $\mathrm{n}=90$ & $\mathrm{n}=56$ & \\
\hline Age, years & $60.02 \pm 10.46$ & $61.88 \pm 11.36$ & $57.04 \pm 8.06$ & 0.003 \\
\hline BMI, $\mathrm{kg} / \mathrm{m}^{2}$ & $24.11 \pm 3.46$ & $24.38 \pm 3.61$ & $23.68 \pm 3.20$ & 0.236 \\
\hline $\mathrm{SBP}, \mathrm{mmHg}$ & $128.77 \pm 17.20$ & $131.37 \pm 13.52$ & $124.60 \pm 21.35$ & 0.020 \\
\hline DBP, $\mathrm{mmHg}$ & $74.48 \pm 9.76$ & $74.17 \pm 9.77$ & $74.99 \pm 9.82$ & 0.621 \\
\hline Smoke, n (\%) & $63(43.15)$ & $37(41.11)$ & $26(46.42)$ & 0.528 \\
\hline Drinking, n (\%) & $25(17.12)$ & $19(21.11)$ & $6(10.71)$ & 0.105 \\
\hline $\mathrm{DM}$ & $59(40.41)$ & $39(43.33)$ & $20(35.71)$ & 0.362 \\
\hline Hypertension & $125(85.62)$ & $78(86.67)$ & $47(83.93)$ & 0.647 \\
\hline Hyperlipidaemia & $76(52.05)$ & $46(51.11)$ & $30(53.57)$ & 0.772 \\
\hline Family history of CVD & $4(2.73)$ & $3(3.33)$ & $1(1.79)$ & 0.578 \\
\hline $\mathrm{TC}, \mathrm{mmol} / \mathrm{L}$ & $3.97 \pm 1.07$ & $3.76 \pm 0.95$ & $4.30 \pm 1.17$ & 0.002 \\
\hline $\mathrm{TG}, \mathrm{mmol} / \mathrm{L}$ & $1.91 \pm 1.38$ & $1.83 \pm 1.11$ & $2.03 \pm 1.74$ & 0.392 \\
\hline LDL-C, $\mathrm{mmol} / \mathrm{L}$ & $2.24 \pm 0.80$ & $2.11 \pm 0.75$ & $2.44 \pm 0.86$ & 0.016 \\
\hline $\mathrm{HDL}-\mathrm{C}, \mathrm{mmol} / \mathrm{L}$ & $0.95 \pm 0.27$ & $0.89 \pm 0.18$ & $1.04 \pm 0.34$ & 0.004 \\
\hline $\mathrm{EF}(\%)$ & $57.42 \pm 9.86$ & $55.49 \pm 10.02$ & $60.54 \pm 8.83$ & 0.002 \\
\hline Aspirin & 115 (78.77) & $73(81.11)$ & $42(75.00)$ & 0.380 \\
\hline Clopidogrel & $104(71.23)$ & $68(75.56)$ & $36(64.29)$ & 0.144 \\
\hline Statin & $107(73.29)$ & $72(80.00)$ & $35(62.50)$ & 0.020 \\
\hline$\beta$-blocker & $82(56.16)$ & $51(56.67)$ & $31(55.36)$ & 0.877 \\
\hline Years of ESRD & $14.36 \pm 8.92$ & $11.45 \pm 8.26$ & $19.04 \pm 7.94$ & $<0.001$ \\
\hline Years of dialysis & $6.19 \pm 5.12$ & $5.29 \pm 3.33$ & $7.62 \pm 6.90$ & 0.021 \\
\hline Years of RT & & & $10.29 \pm 5.74$ & \\
\hline History of PCI & $33(22.60)$ & $16(17.78)$ & $17(30.36)$ & 0.077 \\
\hline
\end{tabular}

Values are given as mean \pm standard deviation, medians with interquartile range or number (\%). BMI, body mass index; CVD, cardiovascular disease; DBP, diastolic blood pressure; DM, diabetes mellitus; ESRD, end-stage renal disease; HDL-C, high-density lipoprotein cholesterol; LDL-C, low-density lipoprotein cholesterol; PCI, percutaneous coronary intervention; RT, renal transplantation; SBP, systolic blood pressure; TC, total cholesterol; $\mathrm{TG}$, triglyceride; $\mathrm{EF}$, ejection fraction.

\subsection{Angiographic characteristics}

CAG findings for patients in this study prior to matching are compiled in Table 3. Patients in the RT group had one-, two-, and three-vessel disease in $50.0 \%, 35.7 \%$, and $14.3 \%$ of cases compared to $35.6 \%, 40.0 \%$ and $24.4 \%$ in dialysis group, respectively, whereas in the dialysis group the incidence of left main disease $(8.9 \%)$ were higher than RT group with scale of $0.0 \%$. The dialysis group also had a significantly higher SYNTAX score (18.09 \pm 7.98$)$ relative to the RT group $(14.79 \pm 10.36)$. A total of $30.4 \%$ and $43.3 \%$ of patients in the RT and dialysis groups, respectively, exhibited moderately to severely calcified lesions. Based on these CAG findings, 23.2\%, 64.3\%, and $12.5 \%$ of RT patients were counseled to undergo medical therapy, percutaneous coronary intervention (PCI), or coronary artery bypass grafting $(\mathrm{CABG})$, respectively, whereas in the dialysis group these percentages were $22.2 \%, 75.6 \%$, and $2.2 \%$, respectively.
CAG findings in study subjects after matching are shown in Fig. 1 and Table 4, which listed the angiographic characteristics after matching. Patients in the RT group had one-, two-, and three-vessel disease in 48.9\%, 37.8\%, and $8.9 \%$ of cases compared to $31.1 \%, 44.4 \%$ and $17.8 \%$ in dialysis group, while rates of left main disease between the dialysis group and RT group are same before matching. Consistently, the SYNTAX score of patients in the dialysis group (17.76 \pm 7.35$)$ remained higher than that of RT group patients (12.57 \pm 5.61$)$. Following matching, 28.9\% and $64.4 \%$ of RT and dialysis group patients exhibited moderately to severely calcified lesions (Fig. 2). Based on these CAG findings, $13.3 \%, 84.4 \%$, and $2.2 \%$ of RT patients were counseled to undergo medical therapy, $\mathrm{PCI}$, or CABG, respectively, whereas in the dialysis group these percentages were $20.0 \%, 71.1 \%$, and $8.9 \%$, respectively (Fig. 3). 
Table 2. Baseline characteristics of involved participants after matching.

\begin{tabular}{|c|c|c|c|c|c|c|c|c|c|}
\hline & \multicolumn{4}{|c|}{ ESRD } & \multicolumn{4}{|c|}{ RT } & \multirow{3}{*}{$p$ value } \\
\hline & Total & CAG & PCI & CABG & Total & CAG & PCI & CABG & \\
\hline & $\mathrm{n}=45$ & $\mathrm{n}=6$ & $\mathrm{n}=38$ & $\mathrm{n}=1$ & $\mathrm{n}=45$ & $\mathrm{n}=9$ & $\mathrm{n}=32$ & $\mathrm{n}=4$ & \\
\hline Age, years & $59.06 \pm 7.41$ & $59.25 \pm 12.63$ & $59.39 \pm 6.59$ & 49 & $59.06 \pm 7.41$ & $59.71 \pm 8.38$ & $59.09 \pm 7.06$ & $57.33 \pm 10.69$ & 1 \\
\hline BMI, $\mathrm{kg} / \mathrm{m}^{2}$ & $24.82 \pm 3.61$ & $23.95 \pm 3.71$ & $24.86 \pm 3.62$ & 22.22 & $25.50 \pm 3.27$ & $28.22 \pm 3.06$ & $25.06 \pm 3.09$ & $22.38 \pm 0.54$ & 0.562 \\
\hline SBP, mmHg & $133.83 \pm 15.00$ & $143.75 \pm 6.24$ & $132.55 \pm 15.55$ & 137.00 & $124.29 \pm 16.90$ & $137.33 \pm 12.50$ & $120.73 \pm 16.58$ & $131.40 \pm 14.08$ & 0.082 \\
\hline DBP, mmHg & $75.91 \pm 8.11$ & $80.75 \pm 8.06$ & $74.65 \pm 8.47$ & 89.00 & $73.36 \pm 10.96$ & $76.33 \pm 10.02$ & $72.55 \pm 11.52$ & $81.75 \pm 7.41$ & 0.422 \\
\hline Smoke, n (\%) & $10(22.22)$ & $3(50.00)$ & $7(18.42)$ & $0(0.00)$ & $23(51.11)$ & $2(22.22)$ & $20(62.50)$ & $1(25.00)$ & 0.004 \\
\hline Drinking, n (\%) & $7(15.56)$ & $2(33.33)$ & $5(46.49)$ & $0(0.00)$ & $0(0.00)$ & $0(0.00)$ & $0(0.00)$ & $0(0.00)$ & 0.006 \\
\hline $\mathrm{DM}$ & $14(31.11)$ & $0(0.00)$ & $14(36.84)$ & $0(0.00)$ & $20(44.44)$ & $4(44.44)$ & $16(50.00)$ & $0(0.00)$ & 0.192 \\
\hline Hypertension & $29(64.44)$ & $4(66.67)$ & 25 (65.79) & $0(0.00)$ & $43(95.56)$ & $8(88.89)$ & $31(96.88)$ & $4(100.00)$ & $<0.001$ \\
\hline Hyperlipidaemia & $20(44.44)$ & $2(33.33)$ & $18(47.37)$ & $0(0.00)$ & $30(66.67)$ & $8(88.89)$ & $19(59.38)$ & $3(75.00)$ & 0.034 \\
\hline Family history of CVD & $1(2.22)$ & $0(0.00)$ & $1(2.63)$ & $0(0.00)$ & $1(2.22)$ & $0(0.00)$ & $1(3.13)$ & $0(0.00)$ & 1 \\
\hline $\mathrm{TC}, \mathrm{mmol} / \mathrm{L}$ & $4.02 \pm 1.08$ & $3.82 \pm 1.05$ & $4.04 \pm 1.10$ & 5.15 & $4.18 \pm 0.86$ & $4.35 \pm 0.81$ & $4.12 \pm 0.88$ & $3.39 \pm 0.45$ & 0.528 \\
\hline $\mathrm{TG}, \mathrm{mmol} / \mathrm{L}$ & $2.13 \pm 1.14$ & $1.80 \pm 0.68$ & $2.20 \pm 1.53$ & 4.73 & $2.01 \pm 2.23$ & $1.83 \pm 0.53$ & $1.54 \pm 0.67$ & $1.48 \pm 0.70$ & 0.820 \\
\hline LDL-C, mmol/L & $2.30 \pm 0.69$ & $2.15 \pm 1.14$ & $2.28 \pm 0.68$ & 2.75 & $2.32 \pm 0.59$ & $2.63 \pm 0.71$ & $2.23 \pm 0.53$ & $0.85 \pm 0.13$ & 0.875 \\
\hline HDL-C, mmol/L & $0.87 \pm 0.18$ & $0.91 \pm 0.08$ & $0.86 \pm 0.19$ & 0.77 & $1.06 \pm 0.31$ & $1.04 \pm 0.24$ & $1.06 \pm 0.34$ & $0.74 \pm 0.31$ & 0.008 \\
\hline $\mathrm{Cr}(\mathrm{mg} / \mathrm{dL})$ & $10.66 \pm 2.54$ & $10.66 \pm 2.54$ & $11.84 \pm 1.56$ & 0.94 & $1.72 \pm 2.09$ & $2.42 \pm 2.64$ & $1.17 \pm 0.32$ & $1.28 \pm 0.34$ & $<0.001$ \\
\hline $\operatorname{eGFR}\left(\mathrm{mL} / \mathrm{min} / 1.73 \mathrm{~m}^{2}\right)$ & $6.37 \pm 2.29$ & $5.16 \pm 0.55$ & $6.21 \pm 2.78$ & 6.14 & $69.24 \pm 3.75$ & $59.69 \pm 75.80$ & $70.98 \pm 29.21$ & $86.98 \pm 22.13$ & $<0.001$ \\
\hline $\mathrm{EF}(\%)$ & $55.18 \pm 10.86$ & $64 \pm 5.57$ & $53.78 \pm 10.91$ & 63 & $62.50 \pm 9.03$ & $54.00 \pm 16.46$ & $63.90 \pm 4.07$ & $59.33 \pm 4.04$ & 0.043 \\
\hline Aspirin & 40 (88.89) & $4(66.67)$ & 35 (92.11) & $1(100.00)$ & $34(75.56)$ & $5(55.56)$ & $26(81.25)$ & $3(75.00)$ & 0.098 \\
\hline Clopidogrel & $34(75.56)$ & $4(66.67)$ & $29(76.32)$ & $1(100.00)$ & 35 (77.78) & $8(88.89)$ & $24(75.00)$ & $3(75.00)$ & 0.083 \\
\hline Statin & 40 (88.89) & $4(66.67)$ & $36(94.74)$ & $0(0.00)$ & $26(57.78)$ & $8(88.89)$ & $15(46.88)$ & $3(75.00)$ & 0.001 \\
\hline$\beta$-blocker & $28(62.22)$ & $4(66.67)$ & $23(60.53)$ & $1(100.00)$ & $30(66.67)$ & $6(66.67)$ & $22(68.75)$ & $2(50.00)$ & 0.660 \\
\hline Years of ESRD & $11.43 \pm 8.04$ & $16.25 \pm 10.40$ & $10.47 \pm 7.44$ & 11.00 & $19.31 \pm 7.83$ & $18.53 \pm 8.08$ & $20.71 \pm 5.27$ & $19.60 \pm 6.67$ & $<0.001$ \\
\hline Years of dialysis & $5.34 \pm 3.83$ & $6.50 \pm 9.00$ & $3.29 \pm 3.06$ & 4.00 & $7.86 \pm 6.22$ & $8.27 \pm 8.59$ & $5.14 \pm 3.61$ & $6.13 \pm 5.67$ & 0.034 \\
\hline Years of RT & - & - & - & - & $10.13 \pm 6.67$ & $10.85 \pm 7.15$ & $9.86 \pm 6.38$ & $9.97 \pm 6.51$ & - \\
\hline
\end{tabular}

Values are given as mean \pm standard deviation, medians with interquartile range or number (\%). BMI, body mass index; CABG, coronary artery bypass grafting; CAG, coronary angiography; CVD, cardiovascular disease; DBP, diastolic blood pressure; DM, diabetes mellitus; eGFR, estimate glomerular filtration rate; ESRD, end-stage renal disease; HDL-C, high-density lipoprotein cholesterol; LDL-C, low-density lipoprotein cholesterol; PCI, percutaneous coronary intervention; RT, renal transplantation; SBP, systolic blood pressure; TC, total cholesterol; TG, triglyceride; $\mathrm{Cr}$, creatinine; EF, ejection fraction. 
Table 3. Angiographic characteristics before matching.

\begin{tabular}{ccccc}
\hline & Total & ESRD & RT & \\
\cline { 2 - 3 } & $\mathrm{n}=146$ & $\mathrm{n}=90$ & $\mathrm{n}=56$ & \\
\hline Number of target vessels & & & & 0.085 \\
One & $42(28.77)$ & $32(35.56)$ & $28(50.00)$ & 0.605 \\
Two & $56(38.36)$ & $36(40.00)$ & $20(35.71)$ & 0.140 \\
Three & $30(20.25)$ & $22(24.44)$ & $8(14.29)$ & 0.006 \\
CTO & $57(39.04)$ & $43(47.78)$ & $14(25.00)$ & 0.022 \\
LM & $8(5.48)$ & $8(8.89)$ & $0(0.00)$ & 0.044 \\
SYNTAX score & $16.82 \pm 9.08$ & $18.09 \pm 7.98$ & $14.79 \pm 10.36$ & \\
Lesion calcification, $\mathrm{n}(\%)$ & & & & 0.117 \\
None to mild & $90(61.64)$ & $51(56.67)$ & $39(69.64)$ & 0.117 \\
Moderate to severe & $56(38.36)$ & $39(43.33)$ & $17(30.36)$ & \\
\hline
\end{tabular}

Values are given as mean \pm standard deviation, medians with interquartile range or number (\%). ESRD, end-stage renal disease; RT, renal transplantation; One, 1-vessel disease; Two, 2-vessels disease; Three, 3-vessels disease; CTO, chronic total occlusion; LM, left main disease.

Number of target vessels

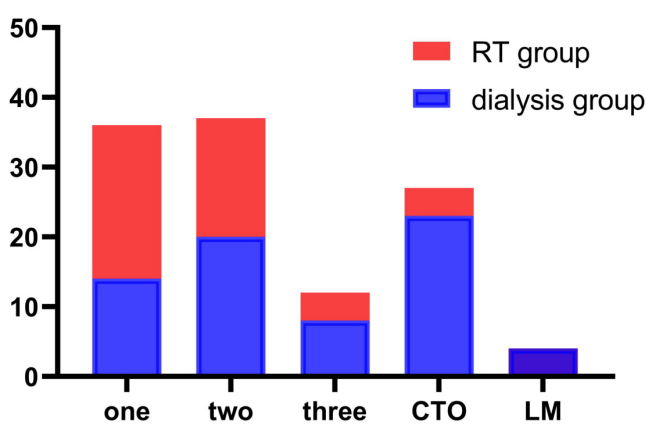

Fig. 1. Angiographic characteristics after matching. RT, renal transplantation; One, 1-vessel disease; Two, 2-vessels disease; Three, 3-vessels disease; CTO, chronic total occlusion; LM, left main disease.

\subsection{Univariate analysis}

In a univariate analysis of the RT group, we found SYNTAX scores to be significantly correlated with history of smoking ( $p=0.009)$, hyperlipidemia $(p=0.004)$, and total ESRD duration $(p<0.001)$ (Table 5).

\subsection{Multivariate analysis}

In a multivariate analysis that incorporated all variables found to be significant in the abovementioned univariate analysis, we found that total ESRD duration $(p<0.001)$ were significantly independently linked with a higher SYNTAX score among RT patients.

\section{Presence of calcification upon examination}

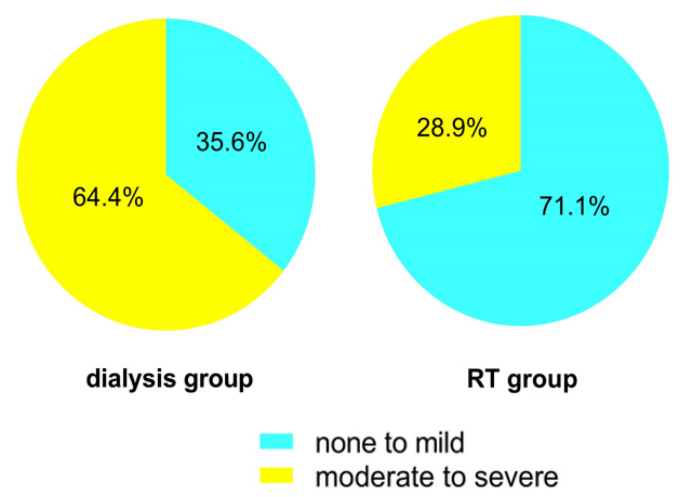

Fig. 2. Calcification in angiography after matching. RT, renal transplantation.

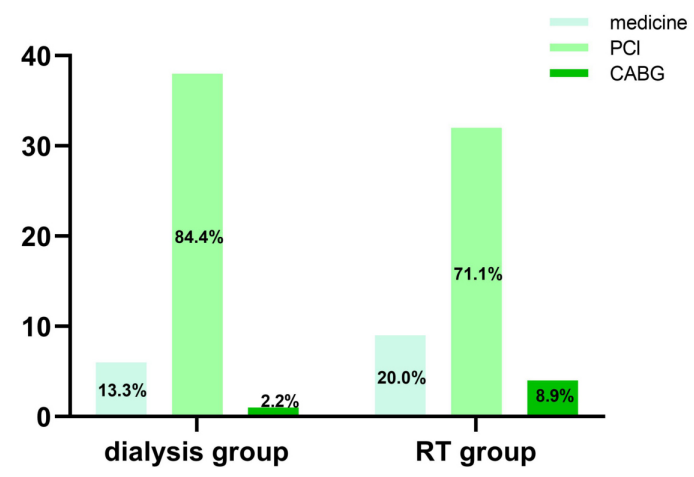

Fig. 3. Therapeutic distribution after matching. PCI, percutaneous coronary intervention; $\mathrm{CABG}$, coronary artery bypass grafting; RT, renal transplantation. 
Table 4. Angiographic characteristics after matching

\begin{tabular}{|c|c|c|c|c|c|c|c|c|c|c|}
\hline & & \multicolumn{4}{|c|}{ ESRD } & \multicolumn{4}{|c|}{ RT } & \multirow{3}{*}{$p$ value } \\
\hline & & Total & CAG & PCI & CABG & Total & CAG & PCI & CABG & \\
\hline & & $\mathrm{n}=45$ & $\mathrm{n}=6$ & $\mathrm{n}=38$ & $\mathrm{n}=1$ & $\mathrm{n}=45$ & $\mathrm{n}=9$ & $\mathrm{n}=32$ & $\mathrm{n}=4$ & \\
\hline History of PCI & & $10(22.22)$ & $2(33.33)$ & $8(21.05)$ & $0(0.00)$ & $8(17.78)$ & $1(11.11)$ & $7(21.88)$ & $0(0.00)$ & 0.598 \\
\hline \multicolumn{11}{|c|}{ Number of target vessels } \\
\hline & One & $14(31.11)$ & $3(50.00)$ & $11(28.95)$ & $0(0.00)$ & $22(48.89)$ & $3(33.33)$ & $12(37.50)$ & $0(0.00)$ & 0.085 \\
\hline & Two & $20(44.44)$ & $0(0.00)$ & $20(52.63)$ & $0(0.00)$ & $17(37.78)$ & $1(11.11)$ & $16(56.25)$ & $0(0.00)$ & 0.520 \\
\hline & Three & $8(17.78)$ & $0(0.00)$ & $7(18.42)$ & $1(100.00)$ & $4(8.89)$ & $0(0.00)$ & $0(0.00)$ & $4(100.00)$ & 0.215 \\
\hline & СТO & $23(51.11)$ & $0(0.00)$ & $17(44.74)$ & $0(0.00)$ & $4(4.44)$ & $0(0.00)$ & $2(6.25)$ & $2(50.00)$ & $<0.001$ \\
\hline & LM & $4(8.89)$ & $0(0.00)$ & $4(10.53)$ & $0(0.00)$ & $0(0.00)$ & $0(0.00)$ & $0(0.00)$ & $0(0.00)$ & 0.041 \\
\hline & SYNTAX score & $17.76 \pm 7.35$ & $16.00 \pm 2.65$ & $18.03 \pm 7.83$ & 34 & $12.57 \pm 5.61$ & $8.67 \pm 7.02$ & $13.63 \pm 5.03$ & $35.26 \pm 5.03$ & 0.03 \\
\hline \multicolumn{11}{|c|}{ Lesion calcification, $\mathrm{n}(\%)$} \\
\hline & None to mild & $16(35.56)$ & $1(16.67)$ & $15(39.47)$ & $0(0.00)$ & $32(71.11)$ & $8(88.89)$ & $23(71.88)$ & $1(25.00)$ & 0.002 \\
\hline & Moderate to severe & $29(64.44)$ & $5(83.33)$ & $23(60.53)$ & $1(100.00)$ & $13(28.89)$ & $1(11.11)$ & $9(28.13)$ & $3(75.00)$ & 0.001 \\
\hline
\end{tabular}

Values are given as mean \pm standard deviation, medians with interquartile range or number (\%). CAG, coronary angiography; CABG, coronary artery bypass grafting; PCI, percutaneous coronary intervention; ESRD, end-stage renal disease; RT, renal transplantation; One, 1-vessel disease; Two, 2-vessels disease; Three, 3-vessels disease; CTO, chronic total occlusion; LM, left main disease. 


\section{Discussion}

To our knowledge, this is the first angiographic study of CAD findings in RT patients in the Chinese Han population. In this analysis, we found that RT patients exhibited lower postoperative CAD severity as assessed via CAG relative to patients undergoing long-term dialysis. This severity analysis was based upon the use of SYNTAX scores, which are commonly used as an objective means of assessing CAD severity [11]. Work by Yan et al. [12] found SYNTAX scores to be negatively correlated with eGFR, emphasizing the relationship between these scores and patient kidney functionality. In the present report, we found that RT patients had a mean SYNTAX score of 12.57 whereas patients undergoing long-term dialysis had a higher score of 17.76. While the score we detected in the dialysis group was lower than that in another previous study (20.6) [13], our RT group score was similar to that reported in this same previous analysis (13.3). We observed higher rates of threevessel or left main disease among patients in the dialysis group, consistent with previous studies. Interestingly, we found that $\mathrm{CAD}$ was less severe on average among patients in the RT group, even though their average ESRD duration (19.3 years) was longer than that of patients in the dialysis group (11.4 years).

Determination of optimal treatments for CAD patients depends in part on determining the degree of lesion calcification in these individuals [14]. Vessel calcification can be challenging to negotiate, while dilation of calcified lesions can be difficult, resulting in suboptimal PCI outcomes even when more advanced interventional strategies are employed [15]. When bypass grafts anastomosed onto calcified vessels, their patency may also be significantly reduced. chronic kidney disease (CKD) patients typical exhibit significant coronary artery calcification [16,17]. Work by Rosas et al, [18] for example, found that even the majority of asymptomatic patients exhibited significant coronary artery calcification at time of RT when examined via computed tomography. In this study, we detected moderately to severely calcified lesions in $28.8 \%$ of RT group patients, with this frequently being slightly below that observed in chronic dialysis patients $(64.4 \% ; p=0.001)$. These calcification rates are lower than those previously reported among RT and ESRD patients in other countries [12,13], suggesting that genetic or environmental factors may account for these differences. As the ESRD duration in RT patients was longer than that in patients in the dialysis group, these results may suggest that transplantation is protective against further vascular calcification.

There have not been sufficient studies conducted to date assessing the optimal strategies for managing $\mathrm{CAD}$ in RT patients. Work by Ferguson et al. [19] found that patients with CKD that underwent PCI or CABG before or after RT had acceptable short- and long-term outcomes. Similarly, research conducted by Herzog et al. [20] detected satisfactory long-term outcomes in RT patients following
Table 5. Correlation between SYNTAX scores and other

\begin{tabular}{lcc}
\multicolumn{3}{c}{ variables. } \\
\hline Variable & $\mathrm{R}$ & $p$ value \\
\hline Age & -0.045 & 0.743 \\
Gender & 0.045 & 0.743 \\
BMI & 0.209 & 0.122 \\
SBP & 0.161 & 0.236 \\
DBP & -0.066 & 0.628 \\
Smoking & 0.347 & 0.009 \\
Drinking & 0.069 & 0.613 \\
DM & 0.128 & 0.347 \\
Hypertension & 0.166 & 0.221 \\
Hyperlipidaemia & 0.434 & 0.001 \\
CVD & 0.108 & 0.429 \\
TC & 0.059 & 0.667 \\
TG & 0.091 & 0.504 \\
LDL-C & -0.036 & 0.795 \\
HDL-C & 0.089 & 0.514 \\
Cr & 0.164 & 0.226 \\
eGFR & 0.005 & 0.972 \\
EF\% & -0.111 & 0.416 \\
Years of ESRD & 0.714 & $<0.001$ \\
Years of dialysis & 0.501 & $<0.001$ \\
Years of RT & 0.426 & 0.001 \\
\hline BMI, Body Mass & &
\end{tabular}

BMI, Body Mass Index; CVD, cardiovascular disease; DBP, diastolic blood pressure; DM, diabetes mellitus; eGFR, estimate glomerular filtration rate; HDL-C, high-density lipoprotein cholesterol; LDLC, low-density lipoprotein cholesterol; RT, renal transplantation; SBP, systolic blood pressure; $\mathrm{Cr}$ : Creatinine; TC, total cholesterol; TG, triglyceride; $\mathrm{EF}$, ejection fraction.

revascularization procedures, with the best outcomes being for patients treated via $\mathrm{CABG}$ using the internal mammary artery. In the present study, CAG results led to the recommendation for RT patients to undergo PCI, CABG, and medical therapy in $84.4 \%, 2.2 \%$, and $13.3 \%$ of cases, respectively, whereas in the dialysis group these rates were $71.1 \%, 8.9 \%$, and $20.0 \%$, respectively. Relative to other similar studies [12,13], we observed a significantly higher rate of patients undergoing PCI and a lower frequency of patients undergoing $\mathrm{CABG}$.

The specific etiology of CAD in RT patients remains to be fully characterized [21]. Immunosuppressive drugs taken by these patients are known to impact CAD-related risk factors such as hypertension, hyperlipidemia, and diabetes incidence [22]. However, RT- and uremia-related factors may also influence the onset of CAD in this patient population [23]. Soveri et al. [24] found that they were able to analyze ALERT trial results in order to develop a means of calculating the cardiovascular risk of RT patients, with the 
resultant risk score being influenced by patient age, diabetes status, creatinine levels, smoking history, time on renal replacement therapy, and history of coronary heart disease. Partially consistent with this, in our small 45 RT patient cohort, we found CAD severity to be significantly correlated with patient smoking status, hyperlipidemia, and total ESRD duration. Multiple previous studies have shown that diabetes, hyperlipidemia, and smoking status are all linked to CAD incidence in the general population and among RT patients [25-28]. CKD is also independently associated with CAD risk through both traditional and uremiaassociated risk factors $[29,30]$. Rates of atherosclerosis are therefore expected to rise significantly with duration of kidney disease, even among functioning graft recipients.

RT patients are known to be at a uniquely high risk of CAD relative to other patient populations $[8,21]$. Although transplantation improved eGFR in treated individuals, it can lead to increased cardiovascular risk, with the metabolic impact of immunosuppressive drug regimens being a major contributor to this risk profile [19]. Even so, RT remains preferable to dialysis in ESRD patients as it is associated with longer survival and better overall quality of life.

There are multiple limitations to the present study. For one, this was a retrospective single-center analysis with a relatively small study population size. In addition, this was a study specifically of RT patients that had undergone CAG due to some clinical indication, and these results may thus not apply to other RT patients. Since the major coronary lesions been treated, the comparison in CAG findings between RT patients with history of PCI and dialysis patients might be very tenuous. In addition, as our dialysis group was designed to match our RT group, findings may not be generalizable to other patients undergoing dialysis. What's more, the different CAD severity is probably not associated with the transplantation because 6 months without dialysis are not an adequate time for an improvement of the CAD. The data may not support a correlation of benefits of renal transplantation and CAD. Lastly, we lack any data to assess clinical outcomes in patients within this study population.

\section{Conclusions}

This study revealed that RT patients had a lower average CAD severity than did age- and sex-matched counterparts undergoing long-term dialysis treatment. Interestingly, this was true even though these RT patients had a longer average ESRD duration than did patients in the dialysis group. These RT patients exhibited high rates of coronary artery calcification, and many were proscribed to undergo PCI based upon CAG findings. Further studies will be needed to determine the optimal clinical treatment strategies from effectively managing CAD in this at-risk patient population.

\section{Abbreviations}

BG, Blood Glucose; BMI, Body Mass Index; CABG, Coronary Artery Bypass Grafting; CAD, Coronary Artery Disease; CAG, Coronary Angiography; CKD, chronic kidney disease; CVD, Cardiovascular Disease; DBP, Diastolic Blood Pressure; DM, Diabetes Mellitus; eGFR, estimate glomerular filtration rate; ESRD, end-stage renal disease; HDL-C, High-Density Lipoprotein Cholesterol; LDL-C, Low-Density Lipoprotein Cholesterol; LM, left main disease; PCI, Percutaneous Coronary Intervention; RT, renal transplantation; SBP, Systolic Blood Pressure; Cr Creatinine; TC, Total Cholesterol; TG, Triglyceride; EF, ejection fraction.

\section{Author contributions}

GYZ, JLW, YYL and YJZ designed the research study. GYZ performed the research. GYZ and JLW analyzed the data. YJZ and YYL revised this paper. All authors read and approved the final manuscript.

\section{Ethics approval and consent to participate}

All subjects gave their informed consent for inclusion before they participated in the study. The study was conducted in accordance with the Declaration of Helsinki, and the protocol was approved by the Ethics Committee of Beijing Anzhen Hospital (approval number: 2022024X).

\section{Acknowledgment}

We thank all our colleagues at the department of Cardiology, Beijing Anzhen Hospital, Capital Medical University.

\section{Funding}

YJZ was supported by National Key Research and Development Program of China (2017YFC0908800), Beijing Municipal Health Commission (Grant No. PXM2020_026272_000002 and Grant No. PXM2020_026272_000014) and Natural Science Foundation of Beijing, China (Grant No. 7212027).

\section{Conflict of interest}

The authors declare no conflict of interest.

\section{References}

[1] Saran R, Robinson B, Abbott KC, Agodoa LYC, Bragg-Gresham $\mathrm{J}$, Balkrishnan R, et al. US renal data system 2018 annual data report: epidemiology of kidney disease in the United States. American Journal of Kidney Diseases. 2019; 73: A7-A8.

[2] Wang XH. International frontier research issue and new progress on renal transplant. Chinese Journal of Organ Transplantation. 2013; 34: 577-581.

[3] Group of Expert Consensus of Contrast Applying in Intervention of Coronary Artery Disease. Expert consensus of contrast applying in intervention of coronary artery disease. Chinese Journal of Cardiovascular Research. 2010; 8: 881-889. 
[4] Li FX, Zhang MR. Research and prevention of contrastinduced nephropathy during perioperative period. Chinese General Practice. 2012; 15: 1311-1313.

[5] Vanrenterghem YFC, Claes K, Montagnino G, Fieuws S, Maes $\mathrm{B}$, Villa M, et al. Risk factors for cardiovascular events after successful renal transplantation. Transplantation. 2008; 85: 209 216.

[6] Kahn MR, Fallahi A, Kim MC, Esquitin R, Robbins MJ. Coronary artery disease in a large renal transplant population: implications for management. American Journal of Transplantation. 2012; 11: 2665-2674.

[7] Israni AK, Snyder JJ, Skeans MA, Peng Y, Maclean JR, Weinhandl ED, et al. Predicting coronary heart disease after kidney transplantation: Patient Outcomes in Renal Transplantation (PORT) Study. American Journal of Transplantation. 2010; 10: 338-353.

[8] Lentine KL, Brennan DC, Schnitzler MA. Incidence and predictors of myocardial infarction after kidney transplantation. Journal of the American Society of Nephrology. 2005; 16: 496-506.

[9] Cai G, Liu W, Lv S, Wang X, Guo Y, Yan Z, et al. Genderspecific associations between atherogenic index of plasma and the presence and severity of acute coronary syndrome in very young adults: a hospital-based observational study. Lipids in Health and Disease. 2019; 18: 99.

[10] Mintz GS, Popma JJ, Pichard AD, Kent KM, Satler LF, Chuang $\mathrm{YC}$, et al. Patterns of calcification in coronary artery disease. a statistical analysis of intravascular ultrasound and coronary angiography in 1155 lesions. Circulation. 1995; 91: 1959-1965.

[11] Sianos G, Morel MA, Kappetein AP, Morice MC, Colombo A, Dawkins K, et al. The SYNTAX Score: an angiographic tool grading the complexity of coronary artery disease. EuroIntervention. 2005; 2: 219-227.

[12] Yan L, Guo L, Zhang F, Gao W. The relationship between kidney function and angiographically-derived SYNTAX score. The Canadian Journal of Cardiology. 2012; 27: 768-772.

[13] Paizis IA, Mantzouratou PD, Tzanis GS, Melexopoulou CA, Darema MN, Boletis JN, et al. Coronary artery disease in renal transplant recipients: an angiographic study. Hellenic Journal of Cardiology. 2020; 61: 199-203.

[14] Hahalis G, Xanthopoulou I, Davlouros P, Mylona P, Tsigkas G, Alexopoulos D. A propensity score-based comparison of flat panel digital detector fluoroscopy versus digital cinefluoroscopy for coronary artery calcium detection. Hellenic Journal of Cardiology. 2012; 53: 205-209.

[15] Généreux P, Redfors B, Witzenbichler B, Arsenault M, Weisz G, Stuckey TD, et al. Two-year outcomes after percutaneous coronary intervention of calcified lesions with drug-eluting stents. International Journal of Cardiology. 2017; 231: 61-67.

[16] Kramer H, Toto R, Peshock R, Cooper R, Victor R. Association between chronic kidney disease and coronary artery calcification: the Dallas Heart Study. Journal of the American Society of Nephrology. 2005; 16: 507-513.

[17] Goodman WG, Goldin J, Kuizon BD, Yoon C, Gales B, Sider $\mathrm{D}$, et al. Coronary-artery calcification in young adults with end- stage renal disease who are undergoing dialysis. The New England Journal of Medicine. 2000; 342: 1478-1483.

[18] Rosas SE, Mensah K, Weinstein RB, Bellamy SL, Rader DJ. Coronary artery calcification in renal transplant recipients. American Journal of Transplantation. 2005; 5: 1942-1947.

[19] Ferguson ER, Hudson SL, Diethelm AG, Pacifico AD, Dean LS, Holman WL. Outcome after myocardial revascularization and renal transplantation: a 25 -year single-institution experience. Annals of Surgery. 1999; 230: 232-241.

[20] Herzog CA, Ma JZ, Collins AJ. Long-term outcome of renal transplant recipients in the United States after coronary revascularization procedures. Circulation. 2004; 109: 2866-2871.

[21] Jardine AG, Gaston RS, Fellstrom BC, Holdaas H. Prevention of cardiovascular disease in adult recipients of kidney transplants. Lancet. 2011; 378: 1419-1427.

[22] Shirali AC, Bia MJ. Management of cardiovascular disease in renal transplant recipients. Clinical Journal of the American Society of Nephrology. 2008; 3: 491-504.

[23] Anastasopoulos N, Dounousi E, Papachristou E, Pappas C, Leontaridou E, Savvidaki E, et al. Cardiovascular disease: Risk factors and applicability of a risk model in a Greek cohort of renal transplant recipients. World Journal of Transplantation. 2017; 7: 49-56.

[24] Soveri I, Holme I, Holdaas H, Budde K, Jardine AG, Fellström B. A cardiovascular risk calculator for renal transplant recipients. Transplantation. 2012; 94: 57-62.

[25] Sukkar L, Talbot B, Jun M, Dempsey E, Walker R, Hooi L, et al. Protocol for the Study of Heart and Renal Protection-Extended Review: Additional 5-Year Follow-up of the Australian, New Zealand, and Malaysian SHARP Cohort. Canadian Journal of Kidney Health and Disease. 2019; 6: 2054358119879896.

[26] Charytan DM, Li S, Liu J, Qiu Y, Herzog CA. Risks of death and graft failure after surgical versus percutaneous coronary revascularization in renal transplant patients. Journal of the American Heart Association. 2013; 2: e003558.

[27] Saran R, Robinson B, Abbott KC, Agodoa LYC, Bragg-Gresham J, Balkrishnan R, et al. US Renal Data System 2018 Annual Data Report: Epidemiology of Kidney Disease in the United States. American Journal of Kidney Diseases. 2019; 73: A7-A8.

[28] Weinrauch LA, Claggett B, Liu J, Finn PV, Weir MR, Weiner $\mathrm{DE}$, et al. Smoking and outcomes in kidney transplant recipients: a post hoc survival analysis of the FAVORIT trial. International Journal of Nephrology and Renovascular Disease. 2018; 11: $155-164$.

[29] Sarnak MJ, Levey AS, Schoolwerth AC, Coresh J, Culleton B, Hamm LL, et al. Kidney disease as a risk factor for development of cardiovascular disease: a statement from the American Heart Association Councils on Kidney in Cardiovascular Disease, High Blood Pressure Research, Clinical Cardiology, and Epidemiology and Prevention. Circulation. 2003; 108: 2154 2169.

[30] Cai Q, Mukku V, Ahmad M. Coronary Artery Disease in Patients with Chronic Kidney Disease: a Clinical Update. Current Cardiology Reviews. 2013; 9: 331-339. 